\title{
Successful Treatment of a Patient with Brentuximab Vedotin-Refractory ALK-Negative Anaplastic Large Cell Lymphoma with Romidepsin
}

\author{
Tatsuro Jo ${ }^{a}$ Takahiro Sakai $^{b}$ Kaori Matsuzakab Haruna Shioyab \\ Hiroo Tominagab ${ }^{b}$ Yohei Kaneko $^{b}$ Shizuka Hayashi $^{b}$ Masatoshi Matsuo ${ }^{a}$ \\ Jun Taguchi $^{a}$ Kuniko Abe ${ }^{c}$ Kazuto Shigematsu ${ }^{c}$ \\ aDepartment of Hematology, Japanese Red Cross Nagasaki Genbaku Hospital, Nagasaki,

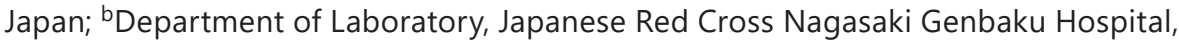 \\ Nagasaki, Japan; 'Department of Pathology, Japanese Red Cross Nagasaki Genbaku \\ Hospital, Nagasaki, Japan
}

\section{Keywords}

Adult T-cell leukemia/lymphoma - Anaplastic large cell lymphoma - Colon cancer - Cytotoxic T lymphocyte $\cdot$ Human T-lymphotropic virus type 1 Tax $\cdot$ Romidepsin

\begin{abstract}
We present the case of a 78-year-old male patient who was diagnosed with anaplastic lymphoma kinase (ALK)-negative, CC chemokine receptor 4 (CCR4)-negative, and CD30-positive anaplastic large cell lymphoma (ALCL). The patient had a past medical history of adult T-cell leukemia/lymphoma and colon cancers that had developed simultaneously approximately 2 years prior to the development of $A L C L$ that were treated with immunochemotherapy and resection, respectively. Initial treatment for $A L C L$ included brentuximab vedotin, an anti-CD30 monoclonal antibody-monomethyl auristatin E conjugate; however, we were unable to achieve a sufficient treatment effect. Romidepsin, an oral histone deacetylase inhibitor, was introduced as salvage chemotherapy; complete remission was attained. Interestingly, a reversal of the CD4/CD8 ratio and a reduction in human T-lymphotropic virus type 1 (HTLV-1) virus load was observed after 2 cycles of immunochemotherapy; the patient experienced upregulation of HTLV-1 Tax-specific cytotoxic T lymphocytes after a herpes zoster infection and the completion of immunotherapy. The immunologic status was maintained from the time of diagnosis through the completion of romidepsin therapy. Our findings indicate that romidepsin can be used safely and effectively to treat ALCL without impairing cellular immunity to HTLV-1.
\end{abstract}




\section{Case Reports in Oncology}

\begin{tabular}{l|l}
\hline Case Rep Oncol 2020;13:1402-1409 \\
\hline DOI: 10.1159/000511111 & $\begin{array}{l}\text { ○ 2020 The Author(s). Published by S. Karger AG, Basel } \\
\text { www.karger.com/cro }\end{array}$ \\
\hline
\end{tabular}

Jo et al.: A Patient Who Overcame ATLL, Colon Cancer, and ALCL

\section{Introduction}

The peripheral T-cell lymphoma (PTCL) known as anaplastic lymphoma kinase (ALK)negative anaplastic large cell lymphoma (ALCL) is notoriously difficult to treat; the 5-year overall survival rate has been reported to be less than 50\% [1]. Currently, no standard therapies have been established for ALK-negative ALCL, although several novel agents, including pralatrexate, romidepsin, brentuximab vedotin (BV), mogamulizumab, and forodesine, have been recently approved for the treatment of PTCLs [2-6]. Adult T-cell leukemia/lymphoma (ATLL) is another form of PTCL; until recently, the prognosis of aggressive ATLL was also quite poor [7]. Human T-lymphotropic virus type 1 (HTLV-1) infection is linked to the development of ATLL; the HTLV-1 protein Tax has been identified as a target of host cellular immunity [7-9].

We here present the case of an elderly male patient who had developed two difficult-totreat PTCLs together with colon cancers over a period of 2 years. Remarkably, he survived and ultimately achieved complete remission (CR).

\section{Case Presentation}

We here report the case of a 78-year-old male patient who presented to our hospital in August 2017 with chief complaints of abdominal pain, nausea, vomiting, and exertional dyspnea. The laboratory data are summarized in Table 1 . At presentation, the patient had an elevated leukocyte count $(10,900 / \mu \mathrm{L})$ with $21.5 \%$ abnormal lymphocytes. Flow cytometry of the peripheral mononuclear cells (PBMCs) revealed that the abnormal lymphocytes (Fig. 1A)

Table 1. Laboratory data at diagnosis with ATLL and ALCL

\begin{tabular}{lll}
\hline & ATLL & ALCL \\
\hline White blood cells, / $\mu \mathrm{L}$ & 10,900 & 11,300 \\
Stab neutrophils, \% & 0.0 & 0.5 \\
Segmented neutrophils, \% & 52.5 & 75.5 \\
Eosinophils, \% & 1.5 & 0.5 \\
Basophils, \% & 0.5 & 1.5 \\
Lymphocytes, \% & 14.5 & 18.5 \\
Abnormal lymphocytes, \% & 21.5 & 0.5 \\
Monocytes, \% & 9.5 & 3.0 \\
Biochemistry & & \\
LDH, U/L & 7,381 & 240 \\
sIL-2R, U/mL & 339,000 & 5,000 \\
Albumin, g/dL & 2.7 & 2.9 \\
Calcium, mg/dL & 9.3 & 8.7 \\
Flow cytometry & PBMCs & PBMCs/LN \\
CD2, \% & 18.2 & Not done/7.4 \\
CD3, \% & 10.9 & $41.2 / 83.8$ \\
CD4, \% & 59.3 & $10.7 / 91.1$ \\
CD8, \% & 3.5 & $30.7 / 2.2$ \\
CD25, \% & 53.6 & Not done/87.2 \\
CD30, \% & 42.4 & Not done/86.1 \\
HTLV-1 provirus DNA, copies/1,000 PBMCs & 119.2 & 6.6 \\
\hline
\end{tabular}

ATLL, adult T-cellleukemia/lymphoma; ALCL, anaplasticlarge cell lymphoma; LDH,lactate dehydrogenase; sIL-2R, soluble interleukin-2 receptor; PBMCs, peripheral blood mononuclear cells; LN, lymph node; HTLV-1, human T-lymphotropic virus type 1. 


\section{Case Reports in Oncology}

\begin{tabular}{l|l}
\hline Case Rep Oncol 2020;13:1402-1409 \\
\hline DOI: 10.1159/000511111 & $\begin{array}{l}\text { @ 2020 The Author(s). Published by S. Karger AG, Basel } \\
\text { www.karger.com/cro }\end{array}$ \\
\hline
\end{tabular}

Jo et al.: A Patient Who Overcame ATLL, Colon Cancer, and ALCL
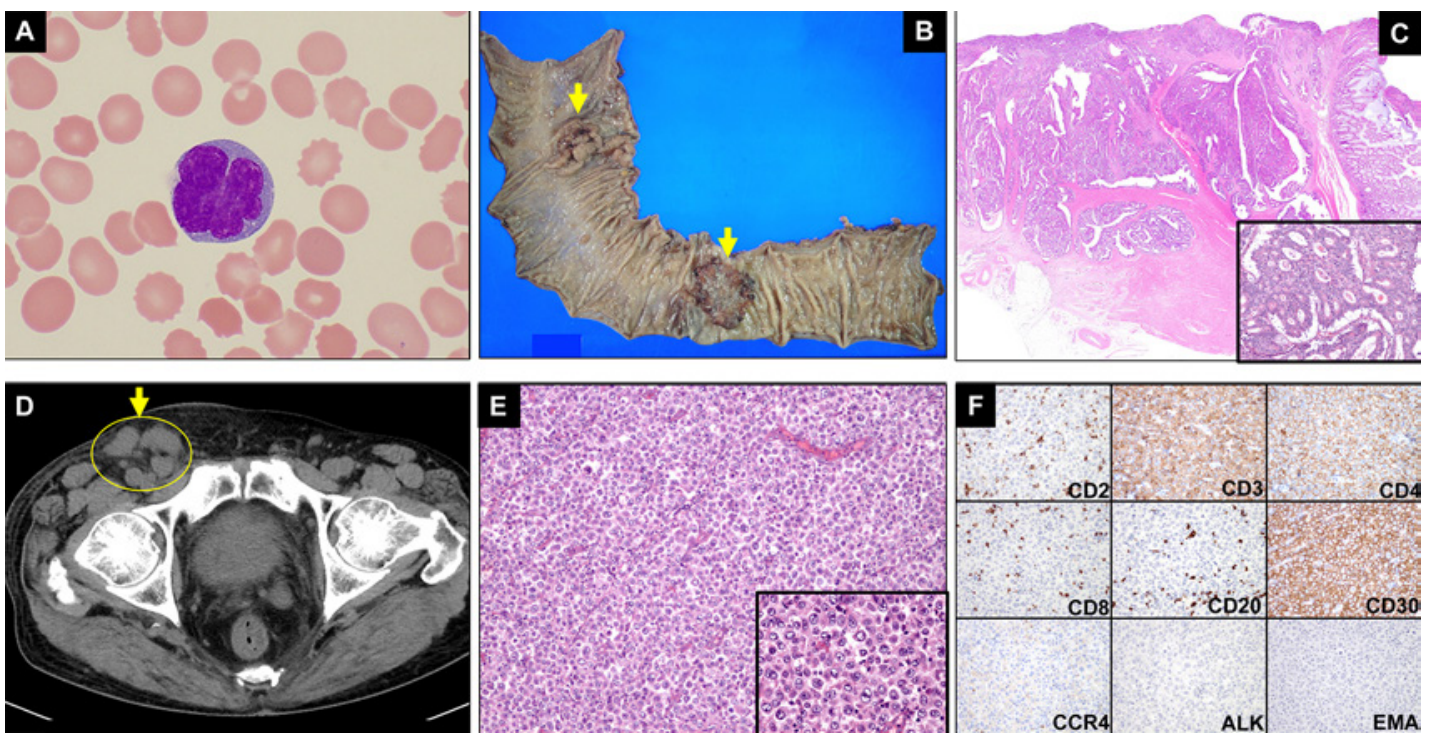

Fig. 1. Clinical images of ATLL, colon cancers, and ALK-negative ALCL. A An abnormal lymphocyte with a flower-shaped nucleus (ATLL cell) detected in a peripheral blood sample from this patient. B The resected tissue included neoplastic tissue from the sigmoid colon and the rectum; the arrows delineate the cancerous regions. C Hematoxylin and eosin (H\&E) staining of the sigmoid colon cancer tissue. The figure inserted in the lower right corner was photographed at a magnification of $\times 100$. D A CT scan revealed lymphadenopathy within the right inguinal region (circle and arrow; June 2019). E H\&E staining of the right inguinal lymph node biopsy specimen ( $\times 100$ magnification). The figure inserted in the lower right corner was photographed at a magnification of $\times 400$. F Immunohistochemical staining of the right inguinal lymph node biopsy specimen with antibodies against antigens as indicated ( $\times 200$ magnification). ATLL, adult T-cell leukemia/lymphoma; ALK, anaplastic lymphoma kinase.

were positive for CD4, CD25, CD30, and CC chemokine receptor 4 (CCR4) and negative for CD2, CD3, and CD8. Tests for anti-HTLV-1 antibody were positive; HTLV-1 provirus DNA was detected at 119.2 copies/1,000 PBMCs. Also notable were lactate dehydrogenase (LDH) and soluble interleukin-2 receptor (sIL-2R) levels, which were markedly elevated at 7,381 U/L and 339,000 U/mL, respectively. A computed tomography (CT) scan revealed left submandibular and hepatic portal lymphadenopathy with hepatosplenomegaly.

Based on these findings, the patient was diagnosed with acute ATLL. The CT scan was also notable for thickening of the sigmoid colon wall; the results from colonoscopy suggested a concurrent diagnosis of colon cancer involving both the rectal and the sigmoid colon. These diagnoses were confirmed pathologically as adenocarcinoma upon colon resection (December 2017; Fig. 1B, C). The patient initially underwent treatment with EPOCH [10] and the antiCCR4 monoclonal antibody mogamulizumab; after 2 rounds of therapy, abnormal lymphocytes were detected only rarely, and the elevated levels of serum LDH had resolved to within normal limits (Fig. 2). Likewise, reversal of the CD4/CD8 ratio was observed after the second cycle of immunochemotherapy; these results suggest that this regimen resulted in upregulation of cytotoxic cellular immunity against ATLL (Fig. 2). The patient then underwent excision of the colon cancers; EPOCH monotherapy was resumed in January 2018. Mogamulizumab was discontinued after the patient had developed a systemic rash; the rash was attributed to mogamulizumab as it emerged after the second cycle of drug therapy. The HTLV-1 provirus load decreased to 20.8 copies/1,000 PBMCs upon resumption of EPOCH; however, a rash secondary to herpes zoster developed on the left anterior chest and back 


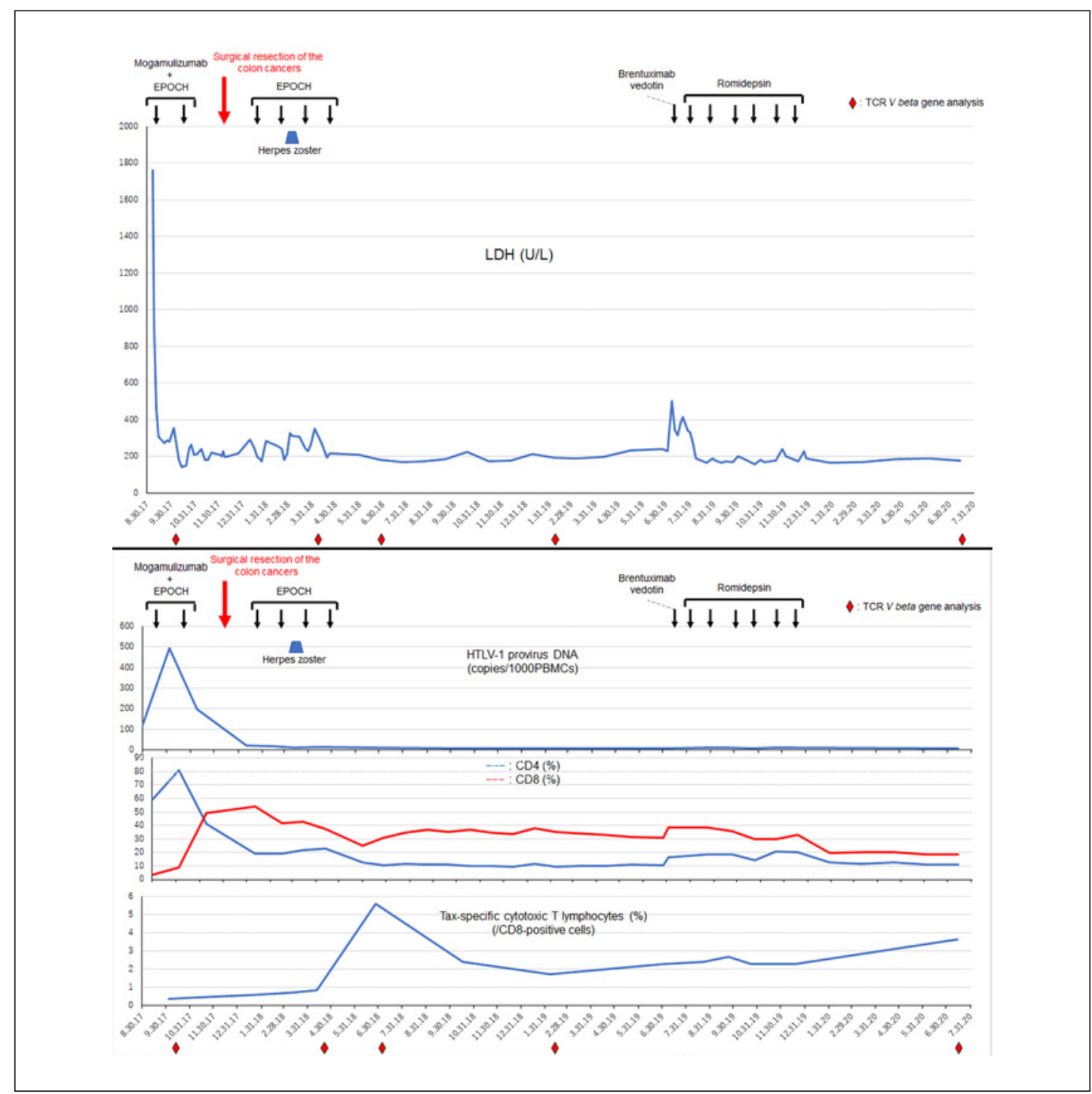

Fig. 2. Changes in levels of serum LDH, HTLV-1 provirus load, and T-cell markers over time. The black arrows indicate each treatment cycle of EPOCH plus mogamulizumab, as well as EPOCH, brentuximab vedotin, and romidepsin monotherapies. The red arrow indicates the timing of the resection of the colon cancers. Upper panel: serum levels of LDH (U/L). Lower panel: the upper part of the lower panel demarcates the HTLV-1 provirus load detected by real-time quantitative polymerase chain reaction over time. The analyses were performed by SRL Corporation (Tokyo, Japan). The middle part of the lower panel includes the percentages of CD4- and CD8-positive cells detected by flow cytometry in samples of patient peripheral mononuclear cells. The lower part of the lower panel reveals the percentages of HTLV-1 Tax-specific CTLs over time. Human leukocyte antigen (HLA)-A*24:02 was detected on patient cells; as such, HLA-A*24:02-restricted Tax tetramer (HLA-A*24:02 HTLV-1 Tax 301-309 Peptide; MBL, Nagoya, Japan) was used for detecting Tax-specific CTLs. The analyses were performed by SRL Corporation. The red rhombi below the graphs indicate time points at which the TCR $V$ beta gene repertoire analysis was evaluated. LDH, lactate dehydrogenase; HTLV-1, human T-lymphotropic virus type 1; CTLs, cytotoxic T lymphocytes.

after the second cycle of monotherapy (Fig. 2). Four cycles of EPOCH monotherapy were administered through April 2018; CR was confirmed by laboratory examinations including a CT scan (data not shown). 


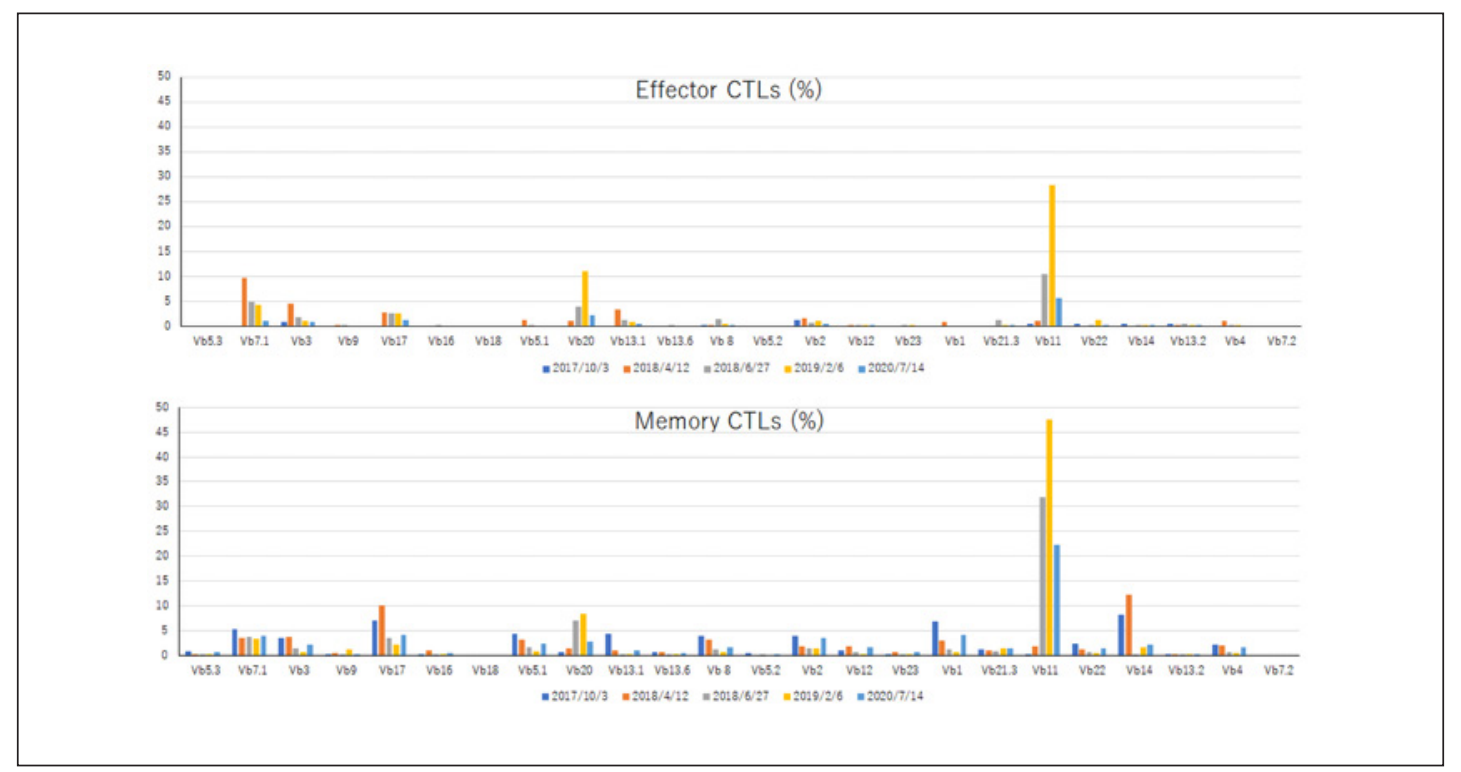

Fig. 3. Analysis of the T-cell receptor $V$ beta gene repertoire. Peripheral blood mononuclear cells were analyzed by flow cytometry using the Beta Mark TCR V $\beta$ Repertoire Kit (Beckman Coulter, Tokyo, Japan) according to the manufacturer's instructions. Effector CTLs (upper panel) were defined as CD8-positive CD27-negative CD45RA-positive cells, and memory CTLs (lower panel) were defined as the CD8-positive CD45RAnegative population. CTLs, cytotoxic T lymphocytes; $\mathrm{Vb}, V$ beta gene.

Of note, cyclophosphamide was excluded from all EPOCH therapies because the patient was advanced in age. Tax-specific cytotoxic T lymphocytes (CTLS) were upregulated after herpes zoster and the completion of immunochemotherapy; this cell population persisted at a high level (the lowest level detected was $1.69 \%$ on February 6, 2019). The reversal of the CD4/CD8 ratio and the very low HTLV-1 provirus load were also maintained (Fig. 2).

The patient became aware of significant right inguinal lymph node swelling approximately 14 months after the completion of the immunochemotherapy for ATLL (June 2019; Fig. 1D). Physical examination and a CT scan also revealed right supraclavicular, bilateral axillar, mediastinal, para-abdominal aortic, and rightiliac lymphadenopathy (data notshown). Initially, we suspected that he was experiencing a recurrence of ATLL. However, the percentage of abnormal lymphocytes remained quite low (0.5\%); likewise, serum levels of LDH and sIL-2R were relatively low (at $240 \mathrm{U} / \mathrm{L}$ and 5,000 U/mL, respectively) compared to those detected in association with ATLL (Table 1). The HTLV-1 provirus load also remained low (6.6 copies/1,000 PBMCs). Flow cytometric analysis of leukocytes from peripheral blood also revealed that the reversal of the CD4/CD8 ratio was maintained. Taken together, these findings suggested the possibility of a distinct neoplasm other than ATLL. A lymph node biopsy led to the diagnosis of ALCL (Fig. 1E). Flow cytometry and immunohistochemical staining of lymph node biopsy tissue revealed that the lymphoma cells were positive for CD3, CD4, and CD30, and negative for CD2, CD8, CD20, CCR4, and ALK (Table 1; Fig. 1F). As CD30 was detected on the cell surface of the lymphoma cells, BV, an anti-CD30 monoclonal antibody conjugate, was introduced as initial treatment. The patient deteriorated on this regimen; serum LDH levels increased to $504 \mathrm{U} / \mathrm{L}$. Therefore, BV was discontinued; therapy with romidepsin, an oral histone deacetylase inhibitor, was initiated. No recurrence of ATLL, colon cancer, or ALK-negative ALCL has been observed for $>8$ months since the completion of romidepsin therapy. The reversal of the CD4/CD8 ratio remained unchanged; likewise, the 
elevated levels of Tax-specific CTLs and the low levels of HTLV-1 provirus load persisted both during and after completion of BV and romidepsin treatment (Fig. 2).

To investigate the clonality of the cellular immunity, T-cell receptor (TCR) $V$ beta gene repertoire analyses were performed using flow cytometry targeting CD8-positive $T$ cells at five distinct time points, as shown in Figure 2. Two effector and two memory CTL clones using TCR $V$ beta 20 and $V$ beta 11 were selectively enhanced after contracting herpes zoster and following the completion of the immunochemotherapy for ATLL; this finding was coincident with upregulation of Tax-specific CTLs (Fig. 2, 3). The effector and memory CTL populations detected with TCR $V$ beta 11 were both markedly elevated (28.2 and $47.6 \%$, respectively) approximately 8 months after the completion of the immunochemotherapy for ATLL; the memory CTLs persisted at a high percentage $(22.2 \%)$ for $>8$ months after the completion of romidepsin therapy (Fig. 2,3).

\section{Discussion and Conclusion}

PTCLs are comparatively difficult-to-treat diseases compared with B-cell lymphomas [1]; as such, the median survival time with either acute or lymphoma-type ATLL is typically less than 10 months [7]. No standard therapies for ALK-negative ALCL or aggressive ATLL have been established, but several agents with distinct antineoplastic mechanisms, including pralatrexate, romidepsin, BV, mogamulizumab, and forodesine, have been used to treat PTCLs [2-6]. In the case of our patient, his ATLL cells were positive for CD4, CD30, and CCR4; by contrast, the ALCL cells were positive for CD4 and CD30, but negative for CCR4 (Table 1; Fig. 1F). An anti-CCR4 monoclonal antibody, mogamulizumab, was used to treat ATLL, in combination with EPOCH chemotherapy; this led to CR. Interestingly, and despite the fact that CD30 was detected on the surface of ALCL cells, BV was no effective therapy in this case. By contrast, mogamulizumab was designed to activate natural killer (NK) cells [5]. Prolonged overall survival was observed in patients with aggressive ATLL treated with mogamulizumab who developed mogamulizumabinduced skin disorders compared to those who did not develop drug-associated skin pathology $[11,12]$. The reversal of the CD4/CD8 ratio occurred immediately after the patient had developed a mogamulizumab-induced rash; these results suggest that mogamulizumab might have activated CTLs with activity against the ATLL cells as well as the capacity to activate NK cells in this patient (Fig. 2). Furthermore, Tax-specific CTLs markedly increased after the patient had experienced herpes zoster via expansion of effector and memory CTLs (Fig. 2).

We previously described the cases of several patients with aggressive ATLL who achieved long-term survival associated with induction of Tax-specific CTLs; prior to the availability of mogamulizumab, these patients also developed reversed CD4/CD8 ratios after infection with herpesviruses including herpes simplex and varicella zoster [8]. These data suggest that herpesvirus infection may lead to the upregulation of antitumor cellular immunity together with cellular immunity against herpes virus infection; another possibility is that antibodybased drugs such as mogamulizumab function by activating host cellular immunity as a primary mechanism of action. BV is among the class of drugs known as antibody-drug conjugates; its pharmacological action is related to its conjugation with monomethyl auristatin $\mathrm{E}$ rather than via activation of host cellular immunity. BV is quite effective for the treatment for Hodgkin lymphoma, which is characterized by a small number of Hodgkin/Reed-Sternberg cells surrounded by T lymphocytes, NK cells, and macrophages. However, BV may be less functional with respect to treatment of PTCLs, especially in cases of a high tumor burden. Actually, BV was not effective for the treatment of ALCL in this case. Since CCR4 antigen was not detected on the surface of the ALCL cells, romidepsin was chosen for a salvage chemotherapy, which ultimately resulted in CR.

\section{Karger's}


Romidepsin is an oral histone deacetylase inhibitor; its effects on the immune system are not fully understood. Both immune cell-suppressive and -enhancing effects have been reported for romidepsin $[13,14]$. In this patient, romidepsin had no impact on the reversal of the CD4/CD8 ratio, although the percentages of both CD4-positive and CD8-positive T lymphocytes decreased after 6 cycles of treatment (Fig. 2). Similarly, romidepsin had no impact on Tax-specific CTLs; the percentage of these cells gradually increased after the completion of romidepsin therapy (Fig. 2). Persistence of Tax-specific CTLs may have contributed to the relapse-free $\mathrm{CR}$ achieved in this case. Taken together, these data suggest that romidepsin therapy does not suppress antitumor cellular immunity over the long term.

In summary, this case report describes a patient who was diagnosed with two distinct and difficult-to-treat PTCLs together with colon adenocarcinoma within a period of a few years. The patient underwent curative excision of the colon cancers; likewise, the immunochemotherapy for ATLL and romidepsin for ALK-negative ALCL facilitated CR. Tax-specific CTLs may contribute to the therapeutic impact on ATLL cells; likewise, subsequent treatment with romidepsin had not impact on the long-term cellular immunity.

\section{Acknowledgement}

The authors thank Ms. Riyo Matsumoto for editing this manuscript.

\section{Statement of Ethics}

Written informed consent for use and disclosure of the protected information was obtained from the patient.

\section{Conflict of Interest Statement}

The authors have no conflicts of interest to declare.

\section{Funding Sources}

There was no funding to prepare this manuscript.

\section{Author Contributions}

T.J. designed the work; acquired, analyzed, and interpreted the data; and wrote this manuscript. T.S., K.M., H.S., H.T., Y.K., S.H., K.A., and K.S. conducted the acquisition and analysis of the data. M.M., J.T., K.A., and K.S. discussed the conception of this manuscript.

\section{Karger'k}




\section{Case Reports in Oncology}

\begin{tabular}{l|l}
\hline Case Rep Oncol 2020;13:1402-1409 \\
\hline DOI: 10.1159/000511111 & $\begin{array}{l}\text { ○ 2020 The Author(s). Published by S. Karger AG, Basel } \\
\text { www.karger.com/cro }\end{array}$ \\
\hline
\end{tabular}

Jo et al.: A Patient Who Overcame ATLL, Colon Cancer, and ALCL

\section{References}

1 Vose J, Armitage J, Weisenburger D. International peripheral T-cell and natural killer/T-cell lymphoma study: pathology findings and clinical outcomes. J Clin Oncol. 2008 Sep;26(25):4124-30.

2 O'Connor OA, Pro B, Pinter-Brown L, Bartlett N, Popplewell L, Coiffier B, et al. Pralatrexate in patients with relapsed or refractory peripheral T-cell lymphoma: results from the pivotal PROPEL study. J Clin Oncol. 2011 Mar;29(9):1182-9.

3 Coiffier B, Pro B, Prince HM, Foss F, Sokol L, Greenwood M, et al. Romidepsin for the treatment of relapsed/ refractory peripheral T-cell lymphoma: pivotal study update demonstrates durable responses. J Hematol Oncol. 2014 Jan;7:11.

4 Pro B, Advani R, Brice P, Bartlett NL, Rosenblatt JD, Illidge T, et al. Brentuximab vedotin (SGN-35) in patients with relapsed or refractory systemic anaplastic large-cell lymphoma: results of a phase II study. J Clin Oncol. 2012 Jun;30(18):2190-6.

5 Ogura M, Ishida T, Hatake K, Taniwaki M, Ando K, Tobinai K, et al. Multicenter phase II study of mogamulizumab (KW-0761), a defucosylated anti-cc chemokine receptor 4 antibody, in patients with relapsed peripheral T-cell lymphoma and cutaneous T-cell lymphoma. J Clin Oncol. 2014 Apr;32(11):1157-63.

6 Maruyama D, Tsukasaki K, Uchida T, Maeda Y, Shibayama H, Nagai H, et al. Multicenter phase 1/2 study of forodesine in patients with relapsed peripheral T cell lymphoma. Ann Hematol. 2019 Jan;98(1):131-42.

7 Ishitsuka K, Tamura K. Human T-cell leukaemia virus type I and adult T-cell leukaemia-lymphoma. Lancet Oncol. 2014 Oct;15(11):e517-26.

8 Jo T, Horio K, Shigematsu K. Cytotoxic T-lymphocyte analysis of aggressive types of adult T-cell leukemia/ lymphoma patients with complete remission after intensive combination chemotherapy. Blood. 2014 Dec; 124(21):1648.

9 Jo T, Kaneko Y, Oishi T, Matsuzaka K, Shioya H, Sakai T, et al. Elevation of memory cytotoxic T lymphocytes, including human $\mathrm{T}$ lymphotropic virus type 1 Tax-specific and hepatitis virus type C-specific cytotoxic $\mathrm{T}$ lymphocytes, in a patient with adult T-cell leukemia/lymphoma and hepatocellular carcinoma. Case Rep Oncol. 2020 Jul;13(2):802-6.

10 Wilson WH, Bryant G, Bates S, Fojo A, Wittes RE, Steinberg SM, et al. EPOCH chemotherapy: toxicity and efficacy in relapsed and refractory non-Hodgkin's lymphoma. J Clin Oncol. 1993 Aug;11(8):1573-82.

11 Jo T, Matsuzaka K, Shioya H, Tominaga H, Sakai T, Kaneko Y, et al. Improved overall survival among newly diagnosed aggressive adult T-cell leukemia/lymphoma patients treated with mogamulizumab plus EPOCH compared to mogamulizumab plus VCAP-AMP-VECP. Blood. 2019 Nov;134(Supplement_1):4048.

12 Yonekura K, Kanzaki T, Gunshin K, Kawakami N, Takatsuka Y, Nakano N, et al. Effect of anti-CCR4 monoclonal antibody (mogamulizumab) on adult T-cell leukemia-lymphoma: cutaneous adverse reactions may predict the prognosis. J Dermatol. 2014 Mar; 41(3):239-44.

13 Kelly-Sell MJ, Kim YH, Straus S, Benoit B, Harrison C, Sutherland K, et al. The histone deacetylase inhibitor, romidepsin, suppresses cellular immune functions of cutaneous T-cell lymphoma patients. Am J Hematol. 2012 Apr; 87(4):354-60.

14 Rosás-Umbert M, Ruiz-Riol M, Fernández MA, Marszalek M, Coll P, Manzardo C, et al. In vivo effects of romidepsin on T-cell activation, apoptosis and function in the BCN02 HIV-1 Kick\&Kill clinical trial. Front Immunol. 2020 Mar;11:418. 